\title{
Single versus Dual Antiplatelet Therapy after Transcatheter Aortic Valve Implantation: A Systematic Review and Meta-Analysis
}

\author{
Xu Hu ${ }^{a} \quad$ Fu-Yao Yang ${ }^{\mathrm{a}}$ Yan Wang $^{\mathrm{a}}$ Yi Zhang ${ }^{\mathrm{a}}$ Mao Chen ${ }^{\mathrm{b}}$ \\ aWest China School of Medicine, West China Hospital, Sichuan University, Chengdu, PR China; \\ ${ }^{b}$ Department of Cardiology, West China Hospital, Sichuan University, Chengdu, PR China
}

\section{Keywords}

Transcatheter aortic valve implantation - Dual antiplatelet therapy · Single antiplatelet therapy · Meta-analysis

\begin{abstract}
Objectives: Although dual antiplatelet therapy (DAPT) with aspirin and clopidogrel is currently recommended in patients after transcatheter aortic valve implantation (TAVI), this practice is not supported by sufficient evidence. This study was performed to compare DAPT to single antiplatelet therapy (SAPT). Methods: An electronic search was completed of PubMed, Embase, Ovid Medline, Web of Science, and Cochrane Central Register of Controlled Trials. Studies comparing DAPT versus SAPT in patients undergoing TAVI were included. Results: Seven studies incorporating 1,445 patients were eligible for the present meta-analysis. There were no significant differences on 30-day all-cause mortality (OR $1.19,95 \% \mathrm{Cl} 0.63-2.25, p=0.593), 6$-month all-cause mortality (OR $1.11,95 \% \mathrm{Cl} 0.49-2.53, p=0.796)$, or 1-year all-cause mortality (OR 1.19, 95\% Cl 0.63- 2.25, $p=0.593$ ). DAPT was associated with an increased risk for 30-day (OR 2.91, 95\% Cl 1.85-4.58, $p<0.01$ ), 6-month (OR 2.54, 95\% Cl 1.26-5.10, $p<$ 0.001 ), and 1-year major and lethal bleeding (OR 2.65, 95\%
\end{abstract}

() 2018 S. Karger AG, Basel
Cl 1.04-6.77, $p=0.041$ ) based on 896, 337, and 583 patients, respectively, compared to SAPT. Conclusion: Compared to SAPT, DAPT was not shown to be superior in reducing thrombotic events, but presented an increased risk of major/lifethreatening bleeding. More randomized controlled trials are needed to provide further evidence.

(c) 2018 S. Karger AG, Basel

\section{Introduction}

Aortic stenosis (AS) is the most common valvular heart disease in developed countries [1]. Aortic valve replacement is an effective therapy for severe AS [2]. Surgical aortic valve replacement (SAVR) leads to good longterm survival and the improvement of symptoms, with a low perioperative risk [2-4]. However, a study showed that $33 \%$ of elderly patients with severe, symptomatic AS were denied surgery because of older age and LV dysfunction [5]. Transcatheter aortic valve implantation (TAVI) has emerged as an alternative choice for high-risk and

X.H. and F.-Y.Y. contributed equally to this study.

\section{KARGER}

E-Mail karger@karger.com

www.karger.com/crd
Mao Chen

Department of Cardiology

West China Hospital, Sichuan University

37 Guoxue Street, Chengdu 610041 (PR China)

E-Mail hmaochen@vip.sina.com 
Fig. 1. Study flow chart of the search strategy.

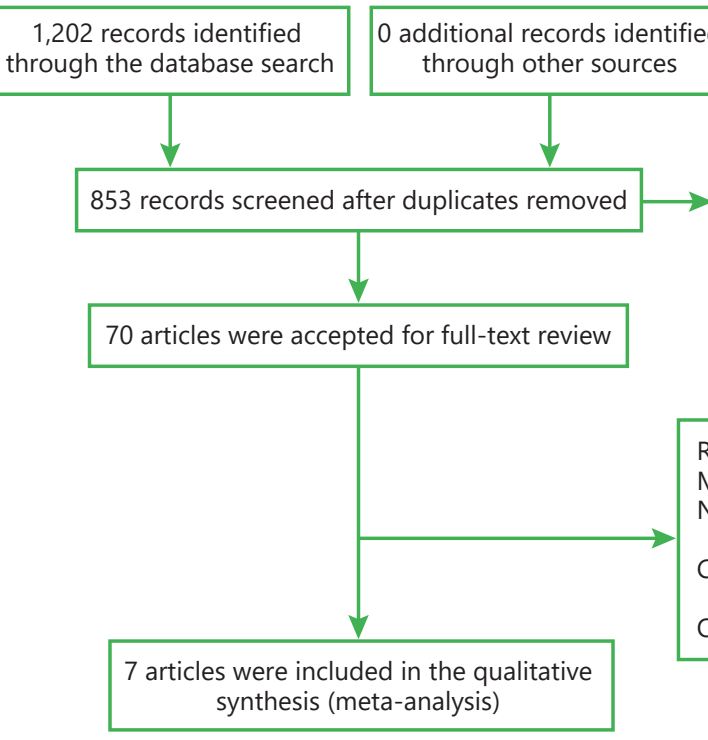

Excluded according to titles and abstracts $(n=673)$ Non-English language $(n=11)$

Review articles $(n=10)$

Meta-analysis ( $n=7$ )

No comparison between

DAPT and SAPT $(n=12)$

Comparison of preprocedural

antiplatelet therapy $(n=5)$

Other exclusions $(n=29)$

inoperable patients with severe AS [6-8]. For intermediate-risk patients with severe AS, TAVI is an alternative choice depending on the patients' procedural risks and preferences [8-10]. TAVI has been shown to be noninferior to SAVR, with reduced kidney injury, atrial fibrillation, and major adverse cardiovascular events in TAVI patients [9-12].

In some studies involving patients undergoing TAVI who had valve thrombosis assessed by computed tomography, the incidence of valve thrombosis varied from 7 to $40 \%[13,14]$. Preventing ischemic events is very important, as this can decrease the risk of atrial fibrillation and device embolization. Therefore, antithrombotic agents are commonly used during and after TAVI. However, due to a lack of evidence, the guidelines do not provide accurate recommendations. For patients without comorbidities, clopidogrel ( $75 \mathrm{mg}$ daily) is recommended for the first 6 months after TAVI in addition to life-long aspirin (75-100 mg daily) [8].

Although dual antiplatelet therapy (DAPT) decreases the risk of thrombotic events, DAPT increases the risk of bleeding compared to single antiplatelet therapy (SAPT) [15]. How to balance the risk of ischemic events and bleeding is still unclear. Some studies have compared the clinical outcomes of DAPT versus SAPT. As presented here, we summarized the relevant research of DAPT versus SAPT with a systematic review and meta-analysis.

\section{Methods}

\section{Search Strategy}

We performed the study according to the Preferred Reporting Items for Systematic Reviews and Meta-Analyses (PRISMA) statement [16]. The PubMed, Embase, Ovid Medline, Web of Science, and Cochrane Central Register of Controlled Trials online databases were searched from inception to May 2017 using terms relating to TAVI (transcatheter, percutaneous, aortic valve replacement, aortic valve implantation, TAVI, TAVR) and treatment (antithrombotic, antiplatelet, platelet aggregation inhibitors, aspirin, acetylsalicylic acid, acid acetylsalicylic, 2-(acetyloxy)benzoic acid, clopidogrel, prasugrel, ticagrelor, $\mathrm{P} 2 \mathrm{Y}$ antagonist, $\mathrm{P} 2 \mathrm{Y}$ receptor antagonist) as key words and Mesh terms. The reference lists of pertinent articles were also screened manually for potentially relevant citations. Two investigators performed the article searches independently, with any disagreement resolved by a third author.

\section{Inclusion and Exclusion Criteria}

In this meta-analysis, we accepted the articles that met the following criteria: (1) population-based studies, (2) randomized controlled trials (RCTs) or observational studies, (3) patients with AS who underwent TAVI, (4) comparison of DAPT with SAPT after TAVI, (5) reported early (30 days or in-hospital) or late ( $\geq 6$ months including early) adverse events (death, bleeding, stroke, etc.). We excluded the following articles: (1) fewer than 20 patients, (2) published duplicate trials, (3) comparison of DAPT with SAPT before TAVI, (4) comparison of DAPT with an oral anticoagulant versus SAPT with an oral anticoagulant, (5) no reporting of the clinical outcomes, (6) articles published in a language other than English. If reported data were duplicated, we only enrolled data from the latest report. 


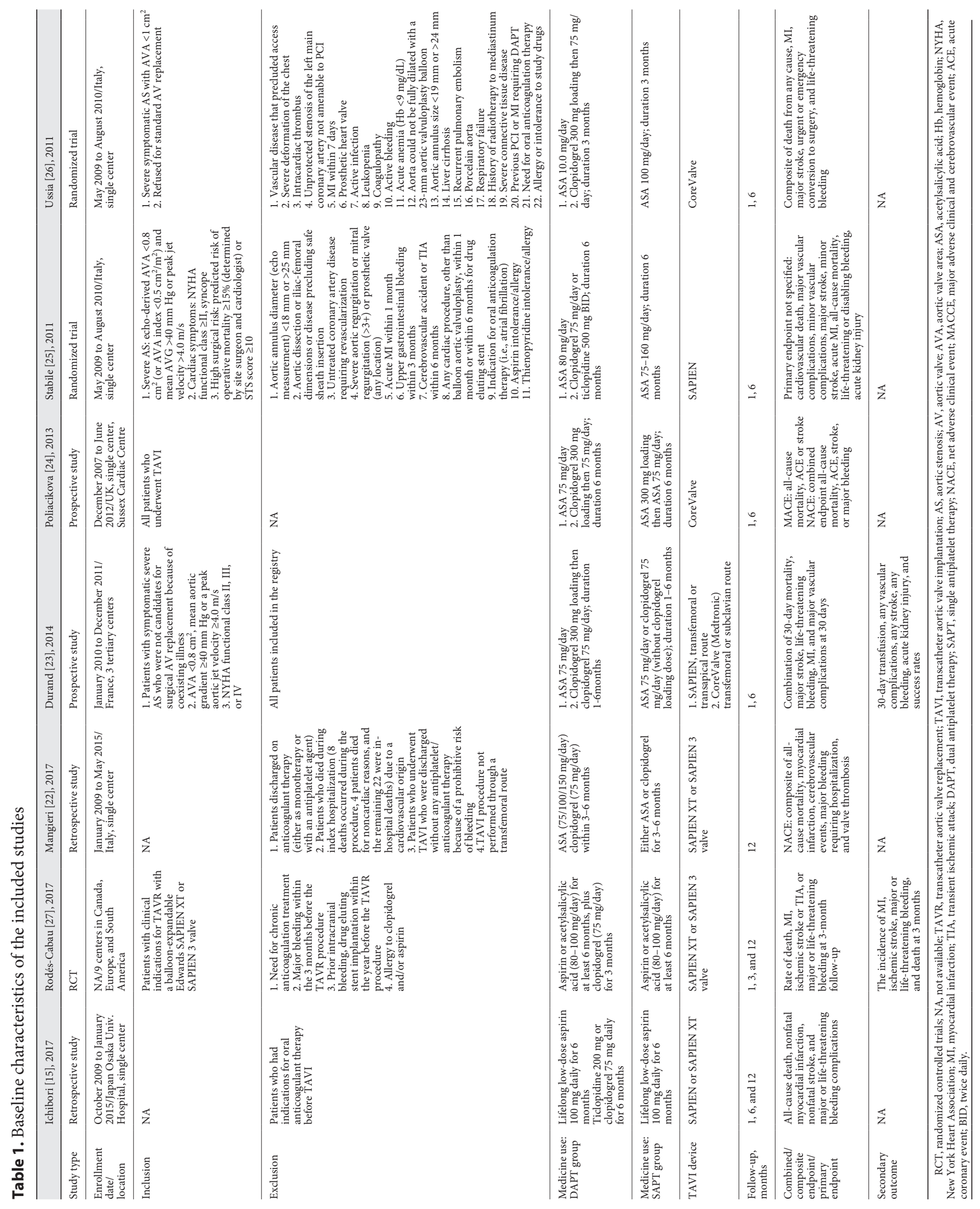


Data Extraction

Two investigators extracted the data independently from the eligible studies, with disagreements resolved by a third author. The name of the first author, year of publication, study type, inclusion and exclusion criteria, duration of follow-up, and patients' baseline data were recorded, as well as factors such as overall mortality, myocardial infarction (MI), bleeding and complications, vascular complications, stroke, etc.

\section{Quality Assessment}

The quality of the relevant articles was assessed by 2 authors. For RCTs, we used the Cochrane Collaboration Risk of Bias Tool to evaluate six bias domains [17]. For case control and cohort studies, we used the Newcastle-Ottawa Quality Assessment Scale to assess quality [18]. Studies were evaluated on the following three aspects: selection, comparability, and exposure/outcome. Studies with a score of 7 or higher were considered high quality. Any disagreements were resolved through consulting a third author.

\section{Outcomes}

The primary endpoints for this study were early (30 days or inhospital) or late ( $\geq 6$ months, including early) all-cause mortality, which can reflect the net effect of thrombotic and bleeding events. The secondary endpoints were MI, valve thrombosis, stroke (major, minor), and transient ischemic attack (TIA), bleeding (lethal, major, minor), and early (30 days or in-hospital) or late ( $\geq 6$ months, including early) vascular complications (major). All clinical events were defined according to the Valve Academic Research Consortium-2 (VARC-2) [19].

\section{Statistical Analysis}

Pooled odds ratios (OR) were acquired using STATA version 12 (StataCorp, College Station, TX, USA) [20]. A two-sided $p$ value $<0.05$ was considered to be the level of statistically significant difference. The Q and $I^{2}$ statistics were calculated to evaluate statistical heterogeneity among the trials. If $I^{2}$ statistics showed $p<$ 0.10 and $I^{2}>50 \%$, a random-effects model was used to estimate the pooled OR [21]. Funnel plots and the Egger regression asymmetry test were conducted to assess publication bias.

\section{Results}

\section{Search Results}

The process of study selection is illustrated in Figure 1. Our search strategy yielded 1,202 articles, 359 of which were duplicated articles. A total of 70 full-text articles remained after screening the titles and abstracts. Finally, a total of 7 articles incorporating 1,445 patients were included in the present study after removing review articles, meta-analyses, and irrelevant articles [15, 22-27].

\section{Characteristics of the Included Articles}

Of the 7 included articles, 3 were RCTs [25-27] and the others were observational articles [16, 22-24]. The mean age was 82.1 years, and $55.6 \%$ of the patients were women. A total of 794 patients were treated with aspirin plus clopidogrel (or ticlopidine) as the DAPT group, and 651 patients were treated with aspirin or clopidogrel as the SAPT group. Four studies completed a 6-month follow-up [23-16], 2 studies completed 12-month follow-up periods $[15,22]$, and 1 study was prematurely stopped [27], reporting the 30-day and 3-month outcomes. Five of the included studies used SAPIEN devices $[15,22-23$, 25,27], while the other 2 used CoreValve devices [24,26]. Detailed characteristics of the articles and patient baseline data are provided in Tables 1 and 2.

\section{Quality Assessment}

The quality assessment of the RCTs using the Cochrane Collaboration Risk of Bias Tool is presented in Table 3 , and the assessment of observational studies using the Newcastle-Ottawa Quality Assessment Scale is shown in Table 4. Four observational studies included in our metaanalysis were considered to be high quality (with scores of $8,8,8$, and 7 ).

\section{All-Cause Mortality}

On the basis of 5 studies incorporating 752 patients, the incidence of 30-day all-cause mortality was found in 22 out of $360(6.1 \%)$ patients in the DAPT group, and in 20 out of $391(5.1 \%)$ in the SAPT group. DAPT had a similar risk for 30-day all-cause mortality, with an OR of 1.19 (95\% CI 0.63-2.25, $p=0.593, I^{2}=0 \%$; Fig. 2 ).

The all-cause mortality rates at 6 months and 1 year were similar. No significant differences for 6-month allcause mortality were noted, with an OR of 1.11 (95\% CI $0.49-2.53, p=0.796, I^{2}=0 \%$; Table 5). Compared to SAPT, DAPT had a similar risk for 1-year all-cause mortality, with an OR of 0.78 (95\% CI $0.38-1.57, p=0.486$, $I^{2}=36.9 \%$; Table 5).

\section{Stroke (Major, Minor) and TIA}

Four studies involving 632 patients reported the incidence of 30-day stoke (major, minor, and TIA). In the DAPT group, the incidence of stroke was $2.8 \%$ (9/300). In the SAPT group, the incidence was $2.6 \%$ (8/332). There was no significant difference between the 2 groups (OR 1.22 , 95\% CI 0.45-3.33, $p=0.698, I^{2}=0 \%$; Fig. 3 ).

The outcomes at 6 months and 1 year between DAPT and SAPT were similar. No significant differences in the incidence of 6-month stroke (major, minor, and TIA) were observed (OR 0.81, 95\% CI 0.21-3.10, $p=$ $0.753, I^{2}=0 \%$; Table 5). Also, DAPT showed a similar incidence for 1-year stroke (major, minor, and TIA; OR $1.58,95 \%$ CI $0.59-4.28, p=0.364, I^{2}=0 \%$; Table 5). 


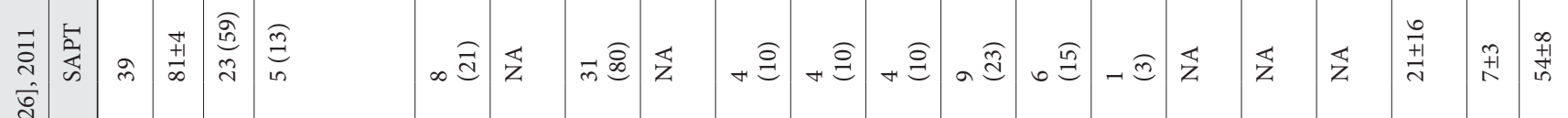

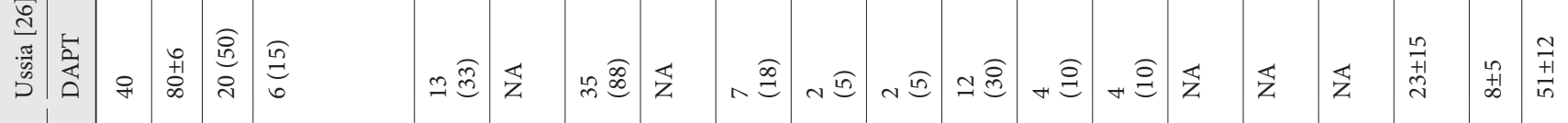

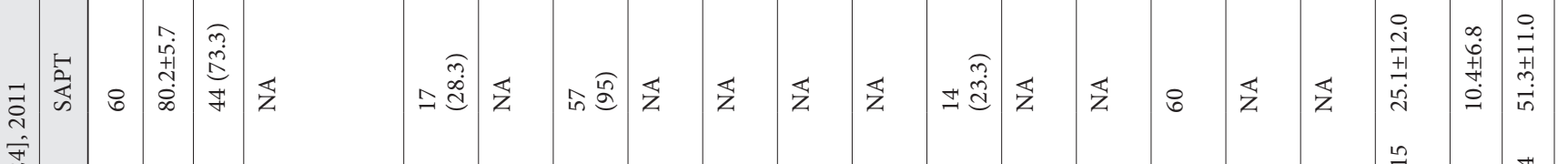

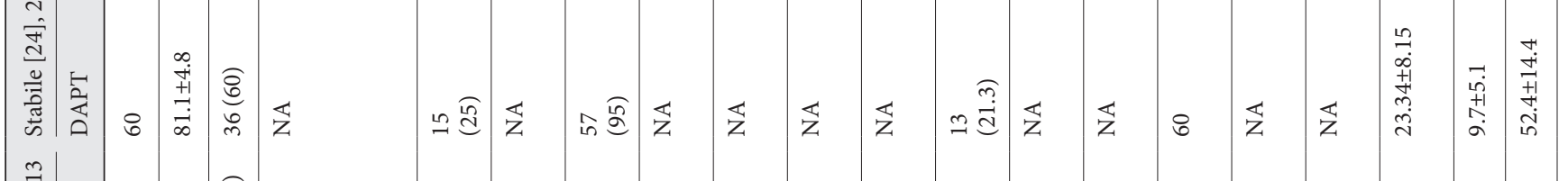

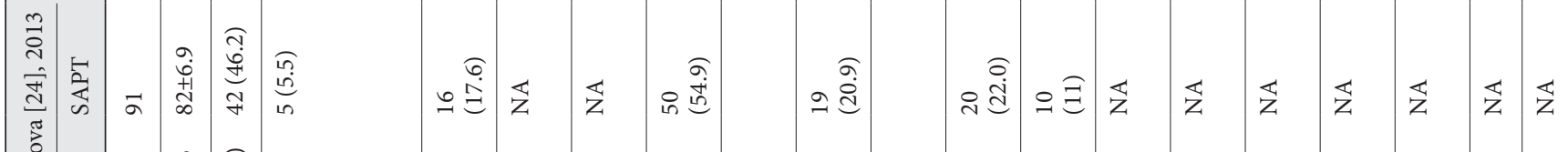

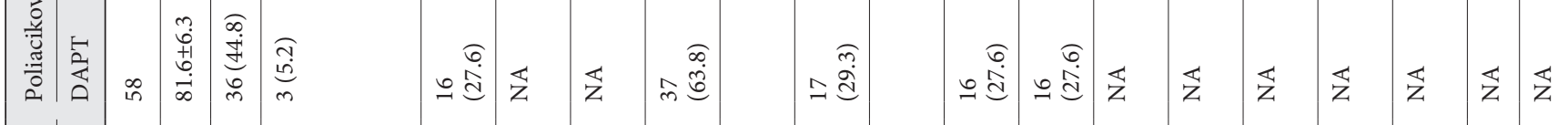

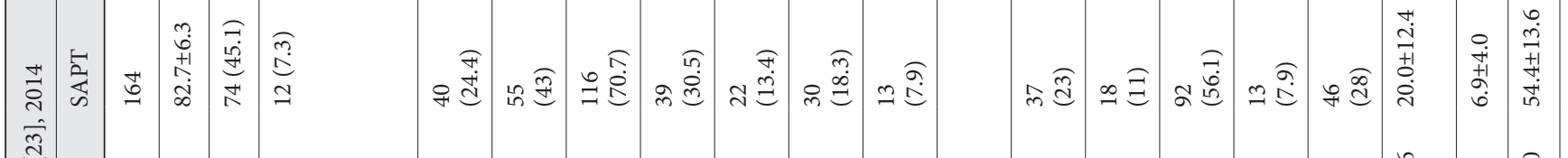

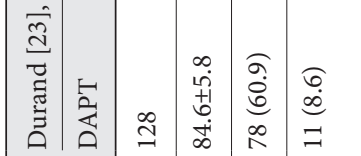

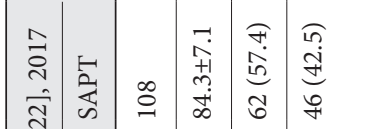

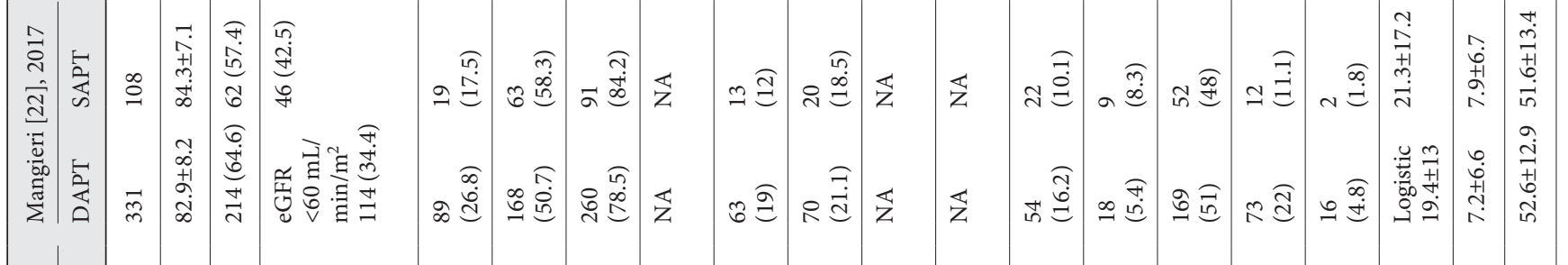

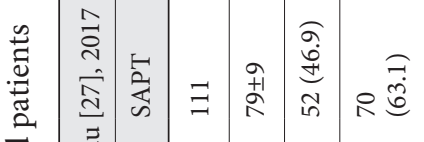

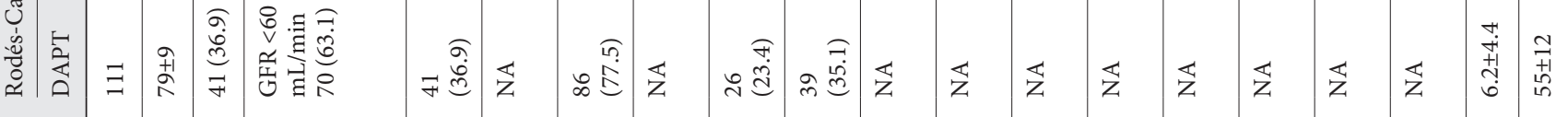

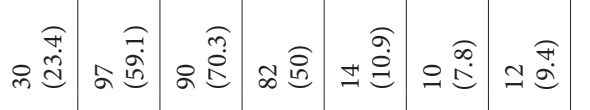

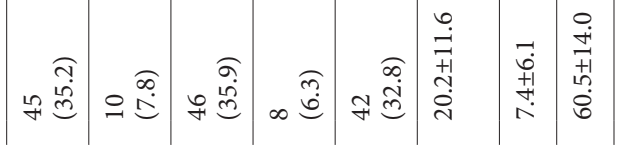

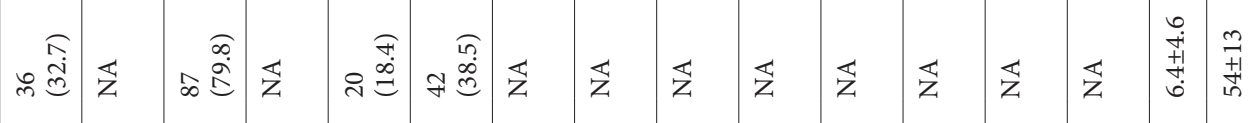

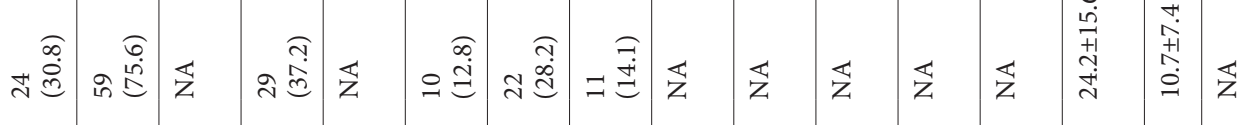

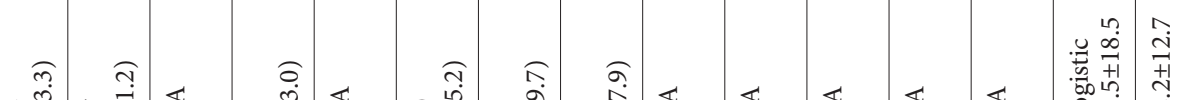

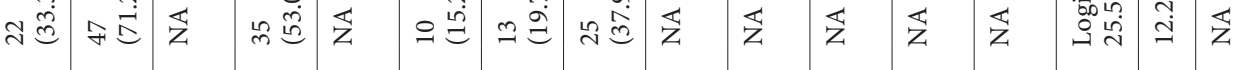

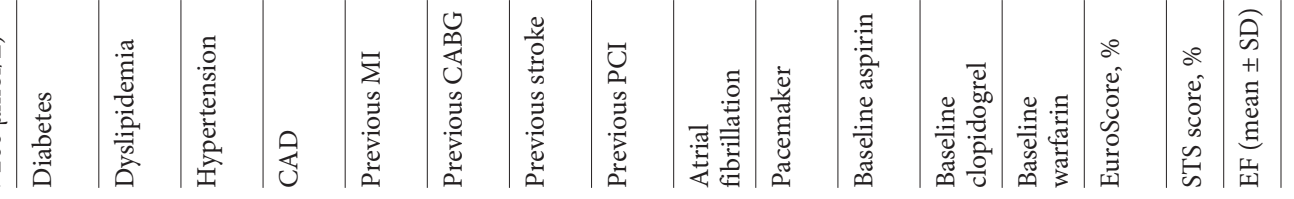




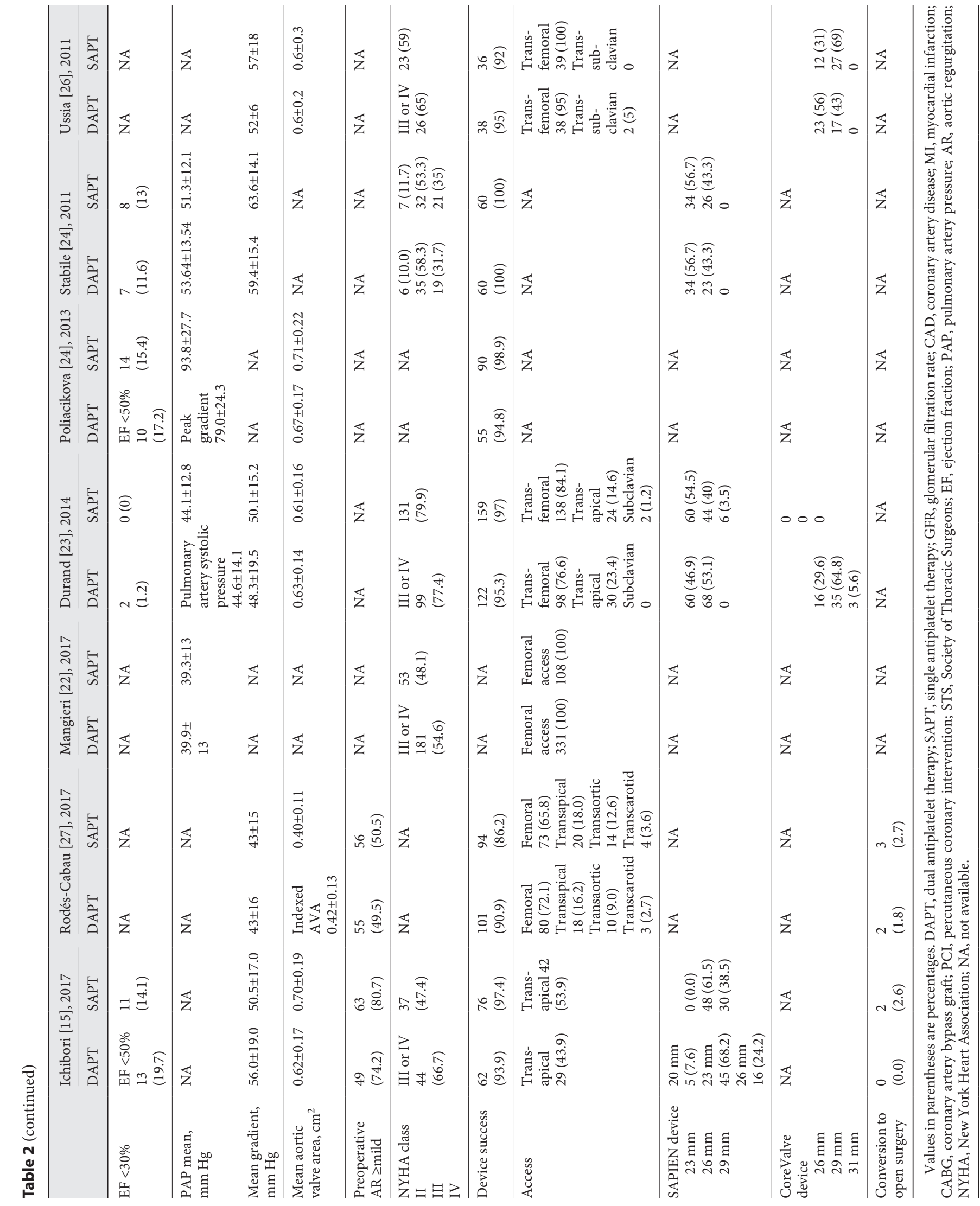


Table 3. Quality assessment of RCTs through the Cochrane Collaboration Risk of Bias Tool

\begin{tabular}{|c|c|c|c|}
\hline Random sequence generation (selection bias) & Low risk & Unclear risk & Unclear risk \\
\hline Blinding of participants and researchers (performance bias) & High risk & Unclear risk & High risk \\
\hline Blinding of outcome assessment (detection bias) & High risk & Unclear risk & Low risk \\
\hline Incomplete outcome data (attrition bias) & High risk & Low risk & Low risk \\
\hline
\end{tabular}

Table 4. Assessment of observational studies through the Newcastle-Ottawa Quality Assessment Scale

\begin{tabular}{|c|c|c|c|c|}
\hline \multicolumn{5}{|l|}{ Selection } \\
\hline Representativeness of exposed cohort & 1 & 1 & 1 & 1 \\
\hline Selection of nonexposed cohort & 1 & 1 & 1 & 1 \\
\hline Ascertainment of exposure & 1 & 1 & 1 & 1 \\
\hline On the basis of the design or analysis & 2 & 1 & 2 & 2 \\
\hline \multicolumn{5}{|l|}{ Outcome } \\
\hline Assessment of outcome & 0 & 0 & 0 & 0 \\
\hline Follow-up long enough for outcomes to occur & 1 & 1 & 1 & 1 \\
\hline Adequacy of follow-up & 1 & 1 & 1 & 1 \\
\hline
\end{tabular}

Table 5. Summary of clinical outcomes (DAPT vs. SAPT)

\begin{tabular}{|c|c|c|c|c|}
\hline 30-day major stroke & 0.99 & $0.27-3.70$ & 0 & 0.99 \\
\hline 30-day TIA & 0.59 & $0.08-4.53$ & 0 & 0.61 \\
\hline 30-day major and minor stroke & 1.16 & $0.39-3.50$ & 0 & 0.79 \\
\hline 6-month all-cause mortality & 1.11 & $0.49-2.53$ & 0 & 0.80 \\
\hline 6-month major and lethal bleeding & 2.54 & $1.26-5.10$ & 0 & 0.009 \\
\hline 1-year major and lethal bleeding & 2.65 & $1.04-6.77$ & 0 & 0.04 \\
\hline
\end{tabular}

DAPT, dual antiplatelet therapy; SAPT, single antiplatelet therapy; TIA, transient ischemic attack.

Furthermore, the stoke incidence (major, minor, and TIA) of the periprocedural period was consistent with the other periods (OR $0.73,95 \%$ CI $0.30-1.78, p=0.484$, $I^{2}=0 \%$; Table 5).
Stroke can be divided into major stroke, minor stroke, and TIA. Compared to SAPT, DAPT showed a similar risk across each of these stroke categories. The detailed statistics are presented in Table 5. 


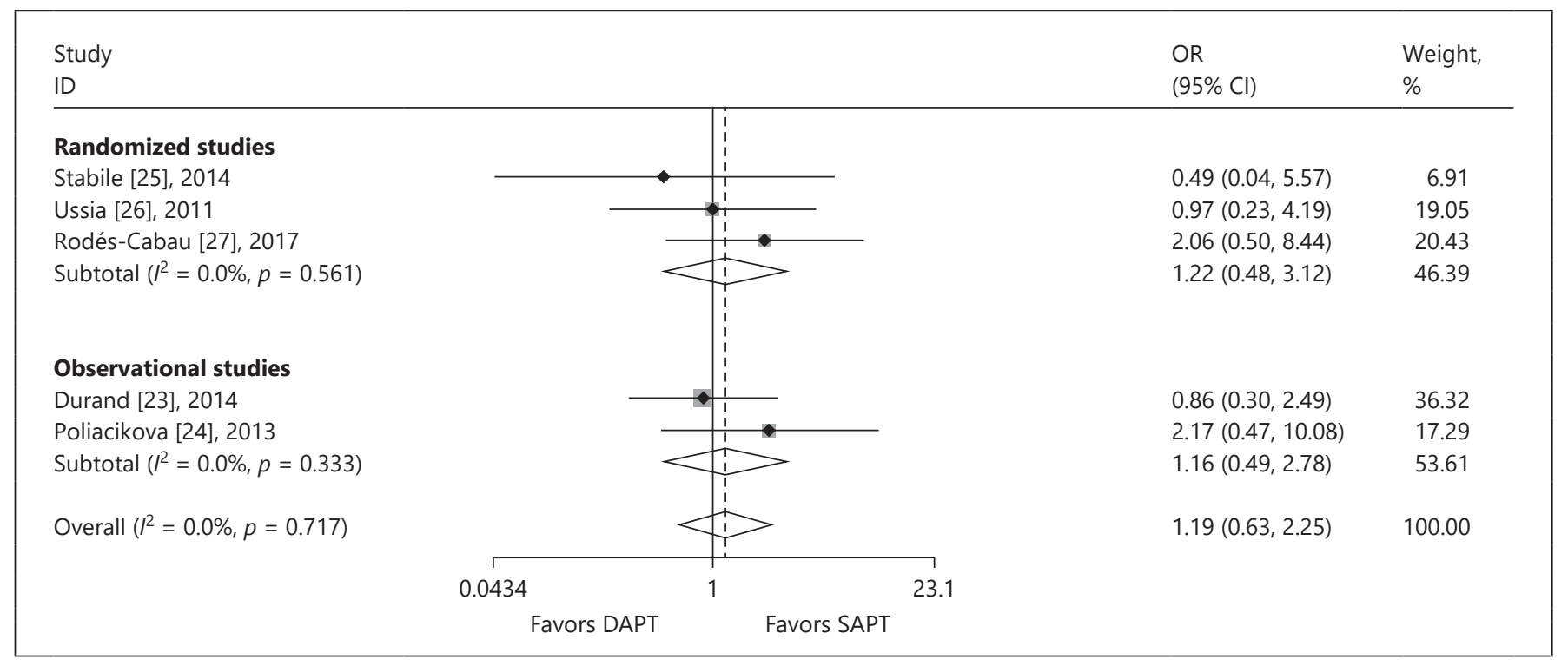

Fig. 2. DAPT versus SAPT for 30-day all-cause mortality.

\begin{tabular}{ll|l}
\hline Study \\
ID
\end{tabular}

Fig. 3. DAPT versus SAPT for 30-day stroke (major, minor, and TIA).

\section{Myocardial Infarction}

Five studies including 752 patients reported the occurrence of 30-day MI. The occurrence rates of 30-day MI in the DAPT and SAPT groups were low, at $0.14 \%(5 / 360)$ versus $1.0 \%$ (4/392). No significant discrepancies in the occurrences of 30-day MI were revealed (OR 1.35, 95\% CI $0.38-4.82, p=0.763, I^{2}=0 \%$; Fig. 4 ).
Bleeding

In 6 studies involving 896 patients, 30-day major and lethal bleeding occurred in 60 of $426(16.2 \%)$ patients in the DAPT group and in 30 of $470(6.4 \%)$ patients in the SAPT group. DAPT had an increased risk for 30-day major and lethal bleeding (OR 2.80, 95\% CI 1.75-4.48, $p<$ $0.001, I^{2}=2.4 \%$; Fig. 5).

Compared to SAPT, DAPT was associated with an increased risk for 6-month major and lethal bleeding (OR 


\begin{tabular}{|c|c|c|c|c|}
\hline $\begin{array}{l}\text { Study } \\
\text { ID }\end{array}$ & & & $\begin{array}{l}\text { OR } \\
(95 \% \mathrm{Cl})\end{array}$ & $\begin{array}{l}\text { Weight, } \\
\%\end{array}$ \\
\hline \multicolumn{5}{|l|}{ Randomized studies } \\
\hline Rodés-Cabau [27], 2017 & & $\frac{i}{1}$ & $4.11(0.45,37.39)$ & 43.40 \\
\hline Stabile [25], 2014 & & $\begin{array}{l}i \\
1 \\
1\end{array}$ & (Excluded) & 0.00 \\
\hline Ussia [26], 2011 & & $i$ & (Excluded) & 0.00 \\
\hline Subtotal & & & $4.11(0.45,37.39)$ & 43.40 \\
\hline \multicolumn{5}{|l|}{ Observational studies } \\
\hline Durand [23], 2014 & $\bullet$ & $\frac{1}{1}$ & $0.49(0.04,5.55)$ & 36.17 \\
\hline Poliacikova [24], 2013 & $\longleftarrow$ & $\frac{1}{1}+1$ & $0.52(0.02,12.87)$ & 20.43 \\
\hline Subtotal $\left(I^{2}=0.0 \%, p=0.984\right)$ & $<$ & $\frac{1}{12}$ & $0.50(0.07,3.47)$ & 56.60 \\
\hline \multirow[t]{3}{*}{ Overall $\left(I^{2}=0.0 \%, p=0.371\right)$} & & 1 & $1.25(0.29,5.35)$ & 100.00 \\
\hline & 0.0207 & 48.4 & & \\
\hline & Favors DAPT & Favors SAPT & & \\
\hline
\end{tabular}

Fig. 4. DAPT versus SAPT for 30-day MI.

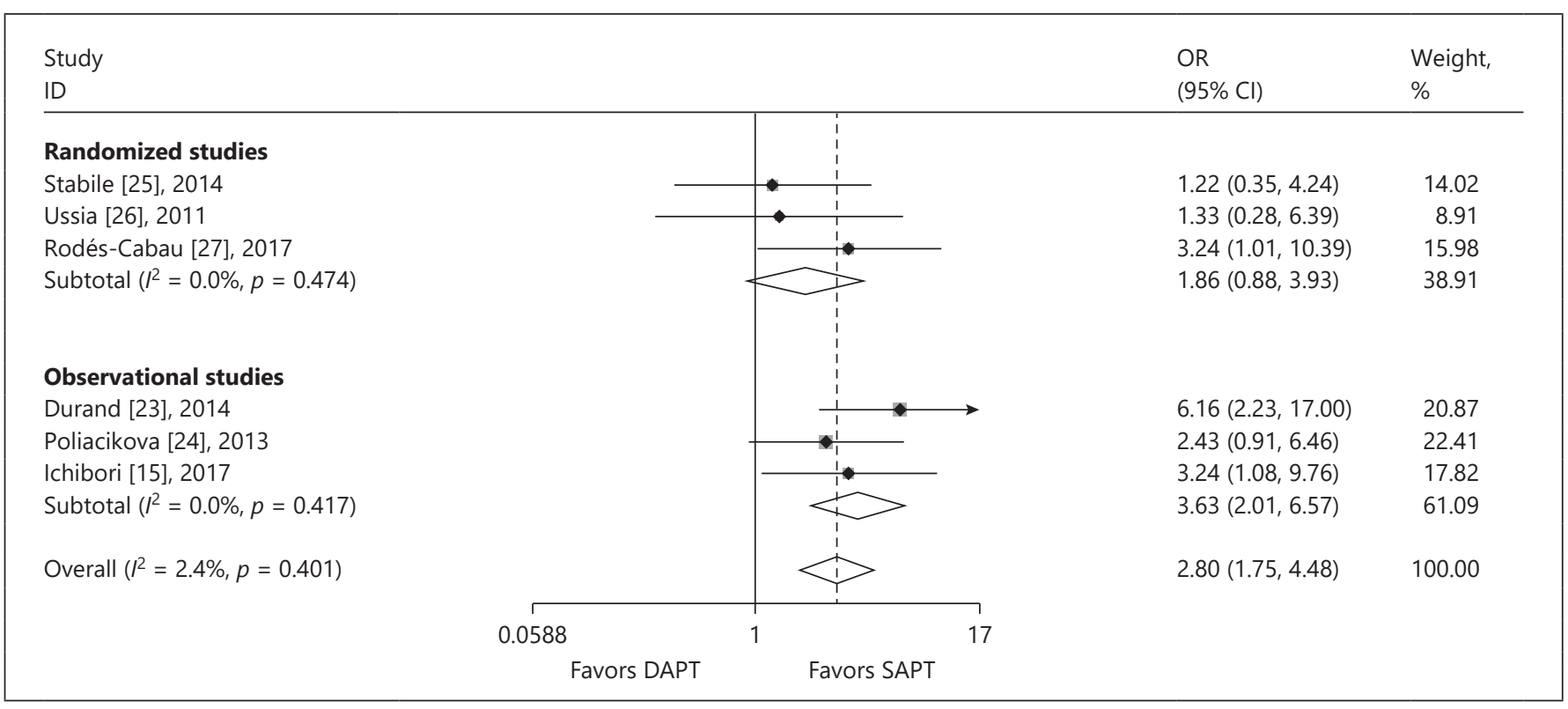

Fig. 5. DAPT versus SAPT for 30-day major and lethal bleeding.

2.54, 95\% CI 1.26-5.10, $p<0.01, I^{2}=0 \%$; Table 5). Patients in the DAPT group had a significantly increased risk of 1-year major and lethal bleeding (OR 2.65, 95\% CI 1.04-6.77, $p=0.041, I^{2}=0 \%$; Table 5). Also, DAPT had a trend towards increased risk for periprocedural major and lethal bleeding (OR 1.95, 95\% CI 0.95-4.01, $p=0.069$, $I^{2}=0 \%$; Table 5).
Three studies including 381 patients reported the incidence of 30-day minor bleeding. In contrast to 30-day major and lethal bleeding, no significant differences in the incidence of 30-day minor bleeding were found (OR 1.01, 95\% CI 0.41-2.49, $p=0.985, I^{2}=0 \%$; Fig. 6). 


\begin{tabular}{|c|c|c|c|c|}
\hline $\begin{array}{l}\text { Study } \\
\text { ID }\end{array}$ & & & $\begin{array}{l}\text { OR } \\
(95 \% \mathrm{Cl})\end{array}$ & $\begin{array}{l}\text { Weight, } \\
\%\end{array}$ \\
\hline Stabile [25], 2014 & & $\longrightarrow$ & $1.53(0.25,9.48)$ & 20.24 \\
\hline Ussia [26], 2011 & & & $0.71(0.15,3.40)$ & 39.91 \\
\hline Durand [23], 2014 & & & $1.05(0.25,4.31)$ & 39.86 \\
\hline \multirow[t]{3}{*}{ Overall $\left(I^{2}=0.0 \%, p=0.821\right)$} & & $=$ & $1.01(0.41,2.49)$ & 100.00 \\
\hline & 0.106 & 9.48 & & \\
\hline & Favors DAPT & Favors SAPT & & \\
\hline
\end{tabular}

Fig. 6. DAPT versus SAPT for 30-day minor bleeding.

\begin{tabular}{l} 
Study \\
ID \\
\hline Stabile [25], 2014
\end{tabular}

Fig. 7. DAPT versus SAPT for 30-day major vascular complications.

\section{Vascular Complications (Major)}

Based on 4 studies incorporating 530 patients, the incidence of 30-day major vascular complications was found to be 22 cases out of $249(8.8 \%)$ in the DAPT group and in 14 out of 281 (5.0\%) in the SAPT group. DAPT had a trend towards increased risk for 30-day major vascular complications but did not reach statistical significance (OR 1.74, 95\% CI 0.87-3.48, $p=0.117, I^{2}=0 \%$; Fig. 7).

\section{Discussion}

In our meta-analysis, we pooled all available studies to compare the outcome of two antiplatelet therapies. The major findings of the present meta-analysis are as follows. (1) There were no significant differences in 30-day, 6-month, and 1-year all-cause mortality between the DAPT and SAPT groups. (2) Regarding ischemic events such as MI and stroke, no significant differences between the 2 groups were found. (3) Concerning bleeding events, we found that DAPT was associated with a 2.9-fold higher risk of 30-day major and lethal bleeding, 2.5-fold higher risk of 6-month major and lethal bleeding, and 2.7-fold higher risk of 1-year major and lethal bleeding. DAPT had a trend towards increased risk for periprocedural major and lethal bleeding. No significant differences in the incidence of 30-day minor bleeding were found. (4) Compared to SAPT, DAPT had a similar risk of 30-day major vascular complications.

We conducted a subgroup analysis between randomized studies and observational studies. No differences in 30 -day major and lethal bleeding, all-cause mortality, stroke (major, minor, and TIA) between the 2 study types were noted. In the randomized studies, there was a trend towards increased risk for 30-day MI in the DAPT group, which was different from the observational studies. In our included studies, some patients used Corevalve devices (self-expandable valve), while some patients used SAPI- 
EN or SAPIEN XT devices (balloon-expandable valve). We excluded 1 study because no detailed information was provided. We found that balloon-expandable valves had a similar risk for 30-day major and lethal bleeding, allcause mortality, and stroke (major, minor, and TIA) compared to self-expandable valves, while discrepancy in 30-day MI between the two kinds of valve was found. Durand et al. [23], Poliacikova et al. [24], and Ussia et al. [26] gave their patients taking clopidogrel a 300-mg preload. According to the subgroup analysis, we found that using a clopidogrel preload had a similar risk for 30-day major and lethal bleeding, all-cause mortality, and stroke (major, minor, and TIA), but not for 30-day MI. In our included articles, 2 reported that no 30-day MI occurred, which may have affected the outcome of different subgroups.

ARTE (aspirin versus aspirin + clopidogrel following TAVI), a latest RCT, may provide powerful information [27]. In this study, patients treated with DAPT had significantly increased risks for 30-day and 3-month major and life-threatening bleeding. However, no significant discrepancies in the incidence of MI and stroke were found. Czerwińska-Jelonkiewicz et al. [28] found that aspirin after TAVI significantly reduced the risk of vascular complications and bleeding with no adverse impact on efficacy endpoints in comparison to other strategies. Sterling et al. [29] revealed a trend towards an increased incidence of major/life-threatening bleeding when comparing DAPT to SAPT, with similar rates of stoke and death. One study also discovered that after TAVI with a balloon-expandable prosthesis, SAPT significantly reduced the rates of major vascular complications and major bleeding without having an impact on valve function [30]. Other studies also revealed similar outcomes [3133]. Therefore, SAPT may be a beneficial option for patients after TAVI, which is also supported by our findings.

Patients undergoing TAVI are usually elderly and may have comorbidities, meaning they may have more clinical symptoms. We considered some mild clinical symptoms, such as minor stroke, TIA, and minor bleeding, which are easily ignored. Compared to SAPT, DAPT had similar risks for these mild clinical symptoms.

In comparison with the studies by Gandhi et al. [34] and Aryal et al. [35], we found some further information, as follows: (1) no discrepancies in the incidence of 30-day minor stroke, TIA, minor bleeding, major vascular complications, 6-month and 1-year clinical outcomes were revealed; (2) the study type, valve, and use of a clopidogrel preload had little effect on 30-day stroke (major, minor, and TIA), all-cause mortality, and major and lethal bleeding, but did impact on 30-day MI.

The current recommendations are based on the few studies that have reported the clinical outcomes of DAPT after TAVI [3], which were empirically determined. TAVI patients are generally old and in a poor condition, which may lead to more dangerous events. The European Society of Cardiology (ESC) and the European Association for Cardio-Thoracic Surgery (EACTS) guidelines did not recommend antiplatelet therapy for the TAVI [2]. The American College of Cardiology (ACC) and American Heart Association (AHA) guidelines recommended 75 mg of clopidogrel daily for the first 6 months after TAVI in addition to life-long aspirin, $75-100 \mathrm{mg}$ daily (classrecommendation, evidence level C) $[3,8]$.

Some studies found that patients undergoing TAVI had valve thrombosis, characterized by reduced leaflet motion, increased mean pressure gradients, and imaging abnormalities [13, 14, 36-38]. These studies demonstrated that oral anticoagulants have a protective effect. Compared to surgical valves, valve thrombosis occurred more commonly in transcatheter valves [37]. Some factors may be considered responsible for valve thrombosis, such as inadequate antiplatelet therapy, patients' hypercoagulability, atrial fibrillation, endothelial injury produced by balloon predilation, and valve malposition, etc. $[13,36-$ 38]. Thus, we should evaluate the condition of patients and their valve function to decide whether oral anticoagulants are needed. According to the guidelines, for patients with a low risk of bleeding, anticoagulation with a vitamin $\mathrm{K}$ antagonist (VKA) is recommended for at least 3 months with an international normalized ratio of 2.5 [8]. Patients with valve thrombosis or atrial fibrillation may need anticoagulation with VKA therapy. AbdulJawad et al. [39] revealed that for patients with VKA therapy, concomitant antiplatelet therapy had no significant difference on stroke or death, while significantly increasing the incidence of major and lethal bleeding. However, the clinical outcomes remain unclear. Banerjee et al. [40] conducted a meta-analysis, finding little evidence of outcomes of patients on anticoagulation after TAVI. There is a need for wider collection of anticoagulation data.

Strokes or TIA are common complications of TAVI. The occurrence of clinical stroke after TAVI averages about 3\% [41]. Patients undergoing TAVI may have a high risk for strokes associated with atrial fibrillation. Chakravarty et al. [37] found that subclinical leaflet thrombosis may be associated with an increased occurrence of stroke or TIA. The occurrence of stroke may lead to a bad prognosis. However, nowadays antithrombotic
62

Cardiology 2018;141:52-65 DOI: $10.1159 / 000490307$
Hu/Yang/Wang/Zhang/Chen 
therapies are not provided accurately. DAPT are recommended empirically, but the efficacy and safety still need further testing.

Bleeding complications are also commonly seen. Major bleeding complications are associated with high mortality and a poor prognosis [42]. The cause of bleeding may be mechanical. Catheters may damage access sites and arteries, which could lead to internal bleeding and vascular complications [41]. Also, patients may have some related diseases, such as arterial calcification, anemia, or gastrointestinal diseases, etc. [43]. Some studies found that patients treated with clopidogrel showed a wide variability in platelet reactivity (PLTR) [44]. One study demonstrated that TAVI-related bleeding occurred during the procedure or in the early postprocedural period, and premedication with clopidogrel enhanced response to clopidogrel early after TAVI, and a significant periprocedural drop in clopidogrel PLTR are considered to increase the risk of TAVI-related bleeding [45]. Hence, PLTR may affect bleeding and ischemic events. Furthermore, patients undergoing TAVI are generally treated with DAPT, which may increase the risk of bleeding. Antiplatelet therapy is necessarily to decrease the risk of ischemic events, meaning bleeding is sometimes inevitable. Bleeding is acceptable, but the most important thing is that it should be controlled well. If bleeding risks becoming lethal, the therapy should be adjusted. How to balance ischemic and bleeding events is still unclear, with further research being needed.

Our meta-analysis reported early (30 days, periprocedure) and late (6 months, 1 year) ischemic and bleeding events. The results provide clinical doctors with the different outcomes of 2 therapies, and open further areas of study for antiplatelet therapy following TAVI. Our study suggests that, when compared to SAPT, DAPT had significantly increased risks for 30-day, 6-month, and 1-year major and lethal bleeding. However, there were no significant differences in MI, stroke, all-cause mortality, minor bleeding, and vascular complications (major) between DAPT and SAPT. SAPT may be a beneficial option. Thus, the efficacy and safety of SAPT requires further research. Regarding patients with comorbidities, the antithrombotic therapy after TAVI also needs more research. One study designed to try to solve these problems is investigating antiplatelet therapy for patients undergoing TAVI (POPular-TAVI, NCT02247128). The POPularTAVI trial is a multicenter open-label RCT which aims to compare the 1-year safety endpoint and net clinical benefit endpoint between aspirin and clopidogrel (cohort A), aspirin monotherapy (cohort $\mathrm{A}$ ), oral anticoagulation and clopidogrel (cohort B), and oral anticoagulation monotherapy (cohort B). It is anticipated that the results of this trial will provide additional information.

\section{Limitations}

There are several limitations to our study. Firstly, 7 studies including 1,445 patients were enrolled, which is a small number, meaning the efficacy and safety cannot be evaluated with sufficient power. TAVI is a new procedure that is unavailable or limited in many hospitals. Secondly, considering that only 2 or 3 studies reported late $(\geq 6$ months including early) clinical outcomes, it was not possible to meaningfully evaluate the mid-term outcomes of the 2 therapies. Thirdly, the procedural approaches reported were different, which may have influenced the clinical outcomes. When the included studies did not report the detailed relevant information, we were unable to perform a subgroup analysis. Lastly, because the patients with atrial fibrillation need oral anticoagulation, we excluded these patients. Although these patients represent a large proportion of those undergoing TAVI, the antithrombotic therapy for these patients is still unclear. It is hoped that the POPular-TAVI trial will offer additional information.

\section{Conclusion}

Compared to SAPT, DAPT appeared to have a similar incidence of MI, stroke, and all-cause mortality, but higher risk for early and mid-term major and lethal bleeding. SAPT may be a beneficial option for patients after TAVI. Further large-scale RCTs are needed to enhance the evidence for antithrombotic strategies in patients undergoing TAVI.

\section{Disclosure Statement}

The authors declare that they have no conflicts of interest to report.

\section{Funding Sources}

This study received no financial support. 


\section{References}

1 Nkomo VT, Gardin JM, Skelton TN, Gottdiener JS, Scott CG, Enriquez-Sarano M. Burden of valvular heart diseases: a population-based study. Lancet. 2006 Sep; 368(9540):1005-11.

2 Vahanian A, Alfieri O, Andreotti F, Antunes MJ, Barón-Esquivias $\mathrm{G}$, Baumgartner $\mathrm{H}$, et al.; Joint Task Force on the Management of Valvular Heart Disease of the European Society of Cardiology (ESC); European Association for Cardio-Thoracic Surgery (EACTS). Guidelines on the management of valvular heart disease (version 2012). Eur Heart J. 2012 Oct;33(19):2451-96.

3 Nishimura RA, Otto CM, Bonow RO, Carabello BA, Erwin JP 3rd, Guyton RA, et al.; American College of Cardiology/American Heart Association Task Force on Practice Guidelines. 2014 AHA/ACC guideline for the management of patients with valvular heart disease: a report of the American College of Cardiology/American Heart Association Task Force on Practice Guidelines. J Am Coll Cardiol. 2014 Jun;63(22):e57-185.

4 Hamm CW, Arsalan M, Mack MJ. The future of transcatheter aortic valve implantation. Eur Heart J. 2016 Mar;37(10):803-10.

5 Iung B, Cachier A, Baron G, Messika-Zeitoun D, Delahaye F, Tornos P, et al. Decision-making in elderly patients with severe aortic stenosis: why are so many denied surgery? Eur Heart J. 2005 Dec;26(24):2714-20.

6 Mack MJ, Leon MB, Smith CR, Miller DC, Moses JW, Tuzcu EM, et al.; PARTNER 1 trial investigators. 5-year outcomes of transcatheter aortic valve replacement or surgical aortic valve replacement for high surgical risk patients with aortic stenosis (PARTNER 1): a randomised controlled trial. Lancet. 2015 Jun; 385(9986):2477-84.

7 Makkar RR, Fontana GP, Jilaihawi H, Kapadia S, Pichard AD, Douglas PS, et al.; PARTNER Trial Investigators. Transcatheter aortic-valve replacement for inoperable severe aortic stenosis. N Engl J Med. 2012 May; 366(18):1696-704.

8 Nishimura RA, Otto CM, Bonow RO, Carabello BA, Erwin JP 3rd, Fleisher LA, et al. 2017 AHA/ACC Focused Update of the 2014 AHA/ACC Guideline for the Management of Patients With Valvular Heart Disease: A Report of the American College of Cardiology/ American Heart Association Task Force on Clinical Practice Guidelines. J Am Coll Cardiol. 2017 Jul;70(2):252-89.

9 Leon MB, Smith CR, Mack MJ, Makkar RR, Svensson LG, Kodali SK, et al.; PARTNER 2 Investigators. Transcatheter or surgical aortic-valve replacement in intermediate-risk patients. N Engl J Med. 2016 Apr;374(17): 1609-20.

10 Thourani VH, Kodali S, Makkar RR, Herrmann HC, Williams M, Babaliaros V, et al. Transcatheter aortic valve replacement versus surgical valve replacement in intermediate- risk patients: a propensity score analysis. Lancet. 2016 May;387(10034):2218-25.

11 Siontis GC, Praz F, Pilgrim T, Mavridis D, Verma S, Salanti G, et al. Transcatheter aortic valve implantation vs. surgical aortic valve replacement for treatment of severe aortic stenosis: a meta-analysis of randomized trials. Eur Heart J. 2016 Dec;37(47):3503-12.

12 Deeb GM, Reardon MJ, Chetcuti S, Patel HJ, Grossman PM, Yakubov SJ, et al.; CoreValve US Clinical Investigators. 3-year outcomes in high-risk patients who underwent surgical or transcatheter aortic valve replacement. J Am Coll Cardiol. 2016 Jun;67(22):2565-74

13 Hansson NC, Grove EL, Andersen HR, Leipsic J, Mathiassen ON, Jensen JM, et al. Transcatheter Aortic Valve Thrombosis: Incidence, Predisposing Factors, and Clinical Implications. J Am Coll Cardiol. 2016 Nov; 68(19):2059-69.

14 Makkar RR, Fontana G, Jilaihawi H, Chakravarty T, Kofoed KF, De Backer O, et al. Possible Subclinical Leaflet Thrombosis in Bioprosthetic Aortic Valves. N Engl J Med. 2015 Nov;373(21):2015-24.

15 Ichibori Y, Mizote I, Maeda K, Onishi T, Ohtani T, Yamaguchi O, et al. Clinical Outcomes and Bioprosthetic Valve Function After Transcatheter Aortic Valve Implantation Under Dual Antiplatelet Therapy vs. Aspirin Alone. Circ J. 2017 Feb;81(3):397-404.

16 Moher D, Liberati A, Tetzlaff J, Altman DG; PRISMA Group. Preferred reporting items for systematic reviews and meta-analyses: the PRISMA statement. PLoS Med. 2009 Jul; 6(7):e1000097.

17 Higgins JP, Altman DG, Gøtzsche PC, Jüni P, Moher D, Oxman AD, et al.; Cochrane Bias Methods Group; Cochrane Statistical Methods Group. The Cochrane Collaboration's tool for assessing risk of bias in randomised trials. BMJ. 2011 Oct;343:d5928.

18 Wells G, Shea B, O'Connell D, et al.; Ottawa Hospital Research Institute. The NewcastleOttawa Scale (NOS) for assessing the quality of nonrandomised studies in meta-analyses. Available from: http://www.ohri.ca/programs/clinical_epidemiology/oxford.asp (accessed September 18, 2014).

19 Kappetein AP, Head SJ, Généreux P, Piazza N, van Mieghem NM, Blackstone EH, et al.; Valve Academic Research Consortium (VARC)-2. Updated standardized endpoint definitions for transcatheter aortic valve implantation: the Valve Academic Research Consortium-2 consensus document (VARC-2). Eur J Cardiothorac Surg. 2012 Nov;42(5):S45-60.

20 Riley RD, Higgins JP, Deeks JJ. Interpretation of random effects meta-analyses. BMJ. 2011 Feb;342:d549.

22 Higgins JP, Thompson SG, Deeks JJ, Altman DG. Measuring inconsistency in meta-analyses. BMJ. 2003 Sep;327(7414):557-60.

22 Mangieri A, Jabbour RJ, Montalto C, Pagnesi M, Regazzoli D, Ancona MB, et al. Single-An- tiplatelet Therapy in Patients with Contraindication to Dual-Antiplatelet Therapy After Transcatheter Aortic Valve Implantation. Am J Cardiol. 2017 Apr;119(7):1088-93.

23 Durand E, Blanchard D, Chassaing S, Gilard M, Laskar M, Borz B, et al. Comparison of two antiplatelet therapy strategies in patients undergoing transcatheter aortic valve implantation. Am J Cardiol. 2014 Jan;113(2):355-60.

24 Poliacikova P, Cockburn J, de Belder A, Trivedi U, Hildick-Smith D. Antiplatelet and antithrombotic treatment after transcatheter aortic valve implantation - comparison of regimes. J Invasive Cardiol. 2013 Oct;25(10):544-8.

25 Stabile E, Pucciarelli A, Cota L, Sorropago G, Tesorio T, Salemme L, et al. SAT-TAVI (single antiplatelet therapy for TAVI) study: a pilot randomized study comparing double to single antiplatelet therapy for transcatheter aortic valve implantation. Int J Cardiol. 2014 Jul;174(3):624-7.

26 Ussia GP, Scarabelli M, Mulè M, Barbanti M, Sarkar K, Cammalleri V, et al. Dual antiplatelet therapy versus aspirin alone in patients undergoing transcatheter aortic valve implantation. Am J Cardiol. 2011 Dec;108(12):1772-6.

27 Rodés-Cabau J, Masson JB, Welsh RC, Garcia Del Blanco B, Pelletier M, Webb JG, et al. Aspirin Versus Aspirin Plus Clopidogrel as Antithrombotic Treatment Following Transcatheter Aortic Valve Replacement With a Balloon-Expandable Valve: The ARTE (Aspirin Versus Aspirin + Clopidogrel Following Transcatheter Aortic Valve Implantation) Randomized Clinical Trial. JACC Cardiovasc Interv. 2017 Jul;10(13):1357-65.

28 Czerwińska-Jelonkiewicz K, Zembala M, Dąbrowski M, Witkowski A, Ochała A, Kochman J, et al. Can TAVI patients receive aspirin monotherapy as patients after surgical aortic bioprosthesis implantation? Data from the Polish Registry - POL-TAVI. Int J Cardiol. 2017 Jan;227:305-11.

29 Sterling LH, Windle SB, Filion KB, Eisenberg MJ. Pharmacological management strategies for stroke prevention following transcatheter aortic valve replacement: a systematic review. Int J Cardiol. 2015 Jul;191:303-11.

30 D’Ascenzo F, Benedetto U, Bianco M, Conrotto F, Moretti C, D'Onofrio A, et al. Which is the best antiaggregant or anticoagulant therapy after TAVI? A propensity-matched analysis from the ITER registry. The management of DAPT after TAVI. EuroIntervention. 2017 Dec;13(12):e1392-400.

31 Turgeon RD, Barry AR. Single vs Dual Antiplatelet Therapy Following Transcatheter Aortic Valve Implantation: A Systematic Review. Clin Cardiol. 2015 Oct;38(10):629-34.

32 Vavuranakis M, Siasos G, Zografos T, Oikonomou E, Vrachatis D, Kalogeras K, et al. Dual or Single Antiplatelet Therapy After Transcatheter Aortic Valve Implantation? A Systematic Review and Meta-Analysis. Curr Pharm Des. 2016;22(29):4596-603. 
33 Ando T, Takagi H, Briasoulis A, Afonso L. Single versus dual anti-platelet therapy post transcatheter aortic valve implantation: a meta-analysis of randomized controlled trials. J Thromb Thrombolysis. 2017 Nov;44(4):44856.

34 Gandhi S, Schwalm JD, Velianou JL, Natarajan MK, Farkouh ME. Comparison of Dualantiplatelet Therapy to Mono-antiplatelet Therapy After Transcatheter Aortic Valve Implantation: Systematic Review and Metaanalysis. Can J Cardiol. 2015 Jun;31(6):77584.

35 Aryal MR, Karmacharya P, Pandit A, Hakim F, Pathak R, Mainali NR, et al. Dual versus single antiplatelet therapy in patients undergoing transcatheter aortic valve replacement: a systematic review and meta-analysis. Heart Lung Circ. 2015 Feb;24(2):185-92.

36 Jose J, Sulimov DS, El-Mawardy M, Sato T, Allali A, Holy EW, et al. Clinical Bioprosthetic Heart Valve Thrombosis After Transcatheter Aortic Valve Replacement: Incidence, Characteristics, and Treatment Outcomes. JACC Cardiovasc Interv. 2017 Apr; 10(7): 686-97.
37 Chakravarty T, Søndergaard L, Friedman J, De Backer O, Berman D, Kofoed KF, et al.; RESOLVE; SAVORY Investigators. Subclinical leaflet thrombosis in surgical and transcatheter bioprosthetic aortic valves: an observational study. Lancet. 2017 Jun;389(10087): 2383-92.

38 De Marchena E, Mesa J, Pomenti S, Marin Y Kall C, Marincic X, Yahagi K, et al. Thrombus formation following transcatheter aortic valve replacement. JACC Cardiovasc Interv. 2015 Apr;8(5):728-39.

39 Abdul-Jawad Altisent O, Durand E, MuñozGarcía AJ, Nombela-Franco L, Cheema A, Kefer J, et al. Warfarin and Antiplatelet Therapy Versus Warfarin Alone for Treating Patients With Atrial Fibrillation Undergoing Transcatheter Aortic Valve Replacement. JACC Cardiovasc Interv. 2016 Aug;9(16): 1706-17.

40 Banerjee K, Poddar K, Mick S, White J, Krishnaswamy A, Johnston D, et al. Meta-Analysis of Usefulness of Anticoagulation After Transcatheter Aortic Valve Implantation. Am J Cardiol. 2017 Nov;120(9):1612-7.

41 Rodés-Cabau J, Dauerman HL, Cohen MG Mehran R, Small EM, Smyth SS, et al. Antithrombotic treatment in transcatheter aortic valve implantation: insights for cerebrovascular and bleeding events. J Am Coll Cardiol. 2013 Dec;62(25):2349-59.
42 Généreux P, Cohen DJ, Williams MR, Mack M, Kodali SK, Svensson LG, et al. Bleeding complications after surgical aortic valve replacement compared with transcatheter aortic valve replacement: insights from the PARTNER I Trial (Placement of Aortic Transcatheter Valve). J Am Coll Cardiol. 2014 Mar;63(11):1100-9.

43 Généreux P, Cohen DJ, Mack M, Rodes-Cabau J, Yadav M, Xu K, et al. Incidence, predictors, and prognostic impact of late bleeding complications after transcatheter aortic valve replacement. J Am Coll Cardiol. 2014 Dec; 64(24):2605-15.

44 Stone GW, Witzenbichler B, Weisz G, Rinaldi MJ, Neumann FJ, Metzger DC, et al.; ADAPTDES Investigators. Platelet reactivity and clinical outcomes after coronary artery implantation of drug-eluting stents (ADAPT-DES): a prospective multicentre registry study. Lancet. 2013 Aug;382(9892):614-23.

45 Czerwińska-Jelonkiewicz K, Witkowski A, Dąbrowski $\mathrm{M}$, et al. The role of platelet reactivity assessment in dual antiplatelet prophylaxis after transcatheter aortic valve implantation. Arch Cardiovasc Dis. 2018 Apr;111(4): 233-245. 